\title{
Kernos
}

Revue internationale et pluridisciplinaire de religion grecque antique

$26 \mid 2013$

Varia

\section{L'oracle de Delphes : quelques mises au point}

\section{Georges Rougemont}

\section{OpenEdition \\ Journals}

Édition électronique

URL : http://journals.openedition.org/kernos/2198

DOI : 10.4000/kernos.2198

ISSN : 2034-7871

\section{Éditeur}

Centre international d'étude de la religion grecque antique

\section{Édition imprimée}

Date de publication : 10 octobre 2013

Pagination : 45-58

ISSN : 0776-3824

Référence électronique

Georges Rougemont, «L'oracle de Delphes : quelques mises au point », Kernos [En ligne], 26 | 2013,

mis en ligne le 31 octobre 2015, consulté le 02 mars 2021. URL : http://journals.openedition.org/

kernos/2198; DOI : https://doi.org/10.4000/kernos.2198 
Kernos 26 (2013), p. 45-58.

\section{L'oracle de Delphes : quelques mises au point}

Résumé : Cet article a pour but d'offrir un bilan clair des connaissances archéologiques actuelles sur l'aménagement intérieur du temple d'Apollon à Delphes, où se déroulait la consultation de l'oracle. Trois temples (au moins) se sont succédé à Delphes. Le premier fut détruit en 548 av. n.è. Le second, achevé avant l'an 500, fut détruit dans les années 370 . Le troisième, terminé vers 330 , vécut jusqu'à l'Antiquité tardive. On ne possède aucune donnée archéologique sur l'aménagement intérieur des deux premiers. Le troisième est un peu moins mal connu, grâce aux travaux récents de P. Amandry et E. Hansen. La cella était divisée en deux parties inégales par une barrière ou une cloison dont on ne sait si elle était assez haute pour arrêter la vue. La seconde partie de la cella, où devait se trouver l'adyton, n'était que partiellement dallée : il faut imaginer un sol en terre battue, mais au même niveau que les dallages, sans local souterrain.

Abstract: The aim of this paper is to offer a clear assessment of the archaeological information available to date regarding the interior fitting of the Apollo temple in Delphi, where the oracle was consulted. Three temples (at least) existed successively in Delphi. The first temple was destroyed in $548 \mathrm{BC}$. The second, completed before 500, was destroyed in the 370s. The third, completed around 330, remained in existence until Late Antiquity. We do not have any archaeological data about the interior fitting of the first two buildings. The third temple is slightly better known, thanks to the recent work of P. Amandry and E. Hansen. The cella was divided into two unequal parts by a barrier or a partition wall; we do not know whether this was high enough to block the view. The second part of the cella, where the adyton must have been located, was only partly paved: we can imagine a clay floor, but at the same level as the paving, without any underground room.

Je ne présente ici aucune conclusion nouvelle, je ne risque aucune hypothèse nouvelle; je n'ai pas découvert le « secret de la Pythie $»^{1}$. Je me contente de quelques mises au point, établies sur la base de connaissances acquises et déjà publiées, parfois depuis longtemps. Ces mises au point portent sur les données archéologiques relatives au lieu de la consultation oraculaire. Mon but est d'offrir aux non spécialistes un bilan des connaissances actuelles, sous une forme aussi claire que possible : je relègue de nombreux détails dans les

\footnotetext{
${ }^{1}$ Chacun connaît la formule d’É. Bourguet, Les ruines de Delphes, Paris, 1914, p. 250 : « La dernière Pythie a emporté son secret. » En 1995, P. AMANDry, Cella, p. $281=$ FD II, 14, p. 79, écrivait encore : "Il est à craindre, comme a écrit Bourguet, que la dernière Pythie n'ait emporté son secret. » À son tour, E. HANSEN, FD II 14 (2010), p. 131, au début de son étude architecturale du temple d'Apollon, prévient : «Le lecteur qui chercherait ici une révélation des secrets de la Pythie serait déçu »; de même M.-Chr. HellmanN, RA (2011), p. 67.
} 
notes et j'évite d'entamer ici la confrontation entre l'archéologie et les textes ${ }^{2}$. Sur chaque problème, j'essaie de tracer une limite entre ce qui, actuellement, peut raisonnablement être considéré comme sûr ou, au moins, comme probable, et ce qui, actuellement, reste incertain ou inconnu. Par nature, cette limite est instable, donc provisoire. Et, comme les données disponibles sont ténues, même les « certitudes » d'aujourd'hui peuvent être contestées demain.

\section{Non pas un, mais trois temples}

Rappelons d'abord un fait bien établi, incontesté depuis des décennies, mais quelquefois perdu de vue. Chacun sait que la consultation de l'oracle de Delphes se déroulait dans le temple d'Apollon; or il y a eu à Delphes non pas un temple d'Apollon, mais trois temples successifs ${ }^{3}$.

1. Le temple parfois appelé « temple du haut-archaïsme ${ }^{4}$ fut détruit par un incendie accidentel la première année de la cinquante-huitième olympiade, soit en 548/7 avant notre ère ${ }^{5}$. Hormis cet événement et sa date, fournie par Pausanias $^{6}$, on ne connaît rien de ce temple ${ }^{7}$, ni la date de sa construction, ni ses dimensions, ni sa structure d'ensemble, ni, à plus forte raison, son aménagement intérieur ${ }^{8}$. Or, si véritablement Crésus consulta l'oracle de Delphes, c'est

\footnotetext{
${ }^{2}$ Ce qui ne va pas sans artifice, évidemment. J'en suis conscient, mais toute confrontation, sur ce sujet, entre l'archéologie et les textes aurait augmenté démesurément le volume du présent exposé. J'espère entreprendre cette confrontation ailleurs.

${ }^{3}$ Ces faits sont dans tous les manuels. Exposés récents : J.-Fr. BOMmELAER, GDS, p. 176184; P. Amandry, Ruine, p. 26-27 = FD II 14, p. 13-14; A. JACQuemin, D. Mulliez, G. Rougemont, ChoixInscrDelphes, "Introduction. 1. Repères chronologiques et historiques ", p. 13-14.

${ }^{4}$ L'expression, conventionnelle, est peu compromettante. Elle serait toutefois contestable si, comme le suggère J.-Fr. BOMMELAER, GDS, p. 183, le temple détruit en 548 datait seulement du premier quart du VIe siècle et avait eu un prédécesseur - dont nous ignorons tout : voir infra note 8.

${ }^{5}$ Ou en 547/6 selon Eusèbe : P. DE La Coste-Messelière, BCH 70 (1946), p. 271 note 1.

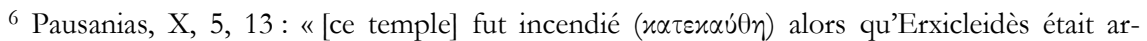
chonte à Athènes, la première année de la cinquante-huitième olympiade, celle où Diognétos de Crotone fut vainqueur [à la course du stade] ». C'est Hérodote, II, 180 qui parle d'un incendie

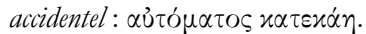

${ }^{7}$ Je veux dire qu'on ne sait rien de sûr: on ne peut faire que des conjectures. Voir la note suivante.

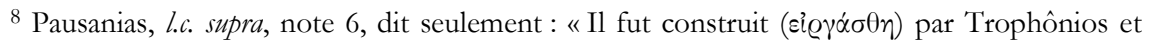

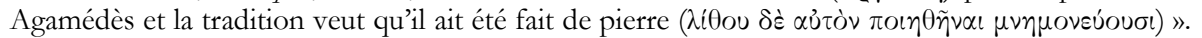
L'Hymne homérique à Apollon, v. 296-297, n'attribue à Trophônios et Agamédès, tous deux fils d'Erginos, que l'installation d'un «seuil de pierre ", $\lambda \dot{\alpha} \iota v o v$ oủoóv, sur des fondations posées par Apollon lui-même, le temple étant construit ensuite, en pierres de taille ( $\varkappa \tau \iota \tau \tau \sigma \tilde{\sigma} \sigma \nu \lambda \dot{\alpha} \varepsilon \sigma \sigma \iota \nu$ ), par

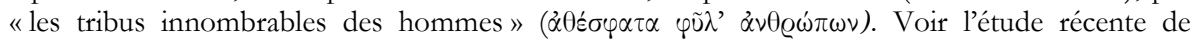
P. BonneChere, Trophonios de Lébadée (2003), p. 71-75 et, plus largement, p. 65-94. De tout cela, il est évidemment impossible de tirer aucune certitude historique. - Quant aux données de l'archéologie, on peut toujours, je crois, souscrire à cette phrase de P. Amandry publiée en 1989 : «Peut-être certains éléments de ce temple [le temple incendié en 548, le plus ancien dont
} 
dans ce temple que la Pythie reçut les envoyés du roi et que furent déposées, en tout cas, certaines de ses plus riches offrandes, endommagées plus tard par l'incendie?.

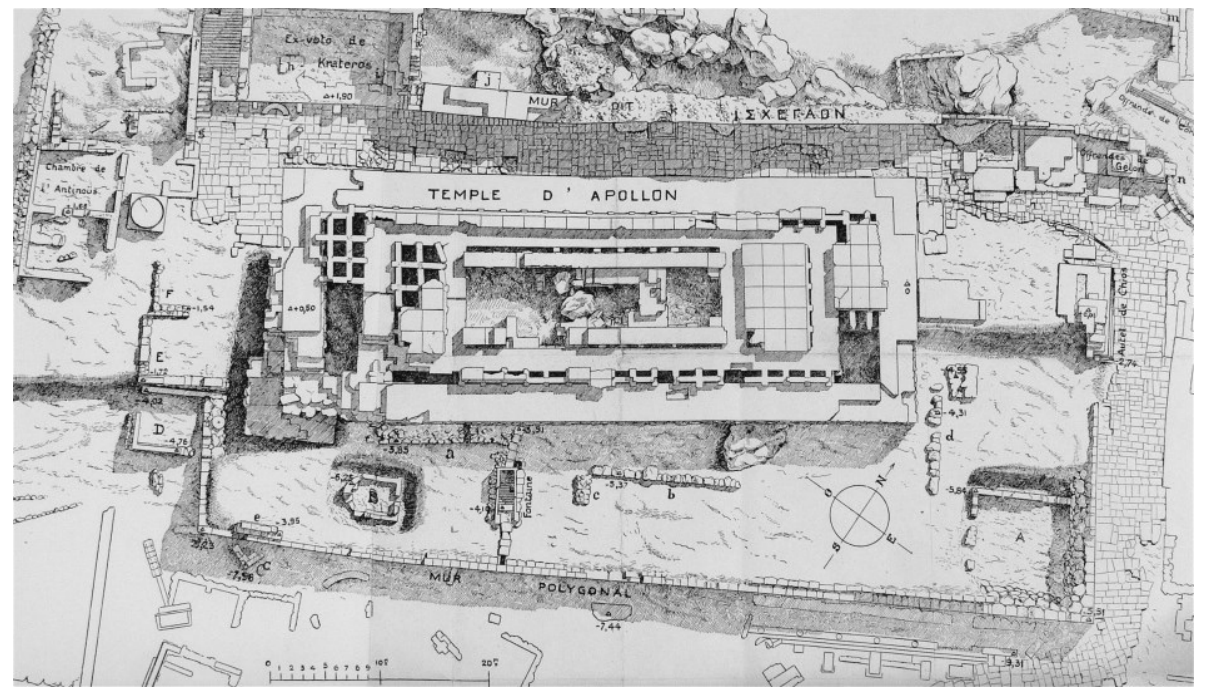

Fig. 1. Plan de la ruine du temple d'Apollon à Delphes au moment de la découverte (F. Courby, FD II. La terrasse du temple [1915], pl. II).

l'existence soit historiquement assurée] subsistent-ils, mais aucun n'a été identifié avec certitude jusqu'à présent » (P. AMANDRY, Ruine, p. $26=F D$ II 14, p. 13). En effet, divers blocs et quelques éléments de toiture sont jugés à la fois antérieurs à 548 et trop gros pour avoir appartenu à un édifice autre qu'un temple, mais toute preuve positive fait défaut : voir J.-Fr. BOMMELAER, GDS, p. 183-184, qui donne la bibliographie antérieure et envisage l'hypothèse de deux temples successifs antérieurs à 548 (voir supra note 4), hypothèse admise comme "probable» par E. HANSEN, BCH 133 (2009), p. 128 note 21, «mais, ajoute ce dernier, nous en savons trop peu pour en tenir compte ici ». E. Hansen, dans l'étude que je viens de citer, présente certains blocs inclus dans la fondation du temple du IV siècle comme «des blocs de remploi provenant du premier temple en pierre ", sans signe de doute, mais sans argumenter; voir aussi la fin de la présente note. - À propos de l'emplacement du temple incendié en 548, F. COURBY, FD II. La terrasse du temple, p. 109, écrivait: «Il est hors de doute que le temple primitif se dressait sur le même emplacement que ceux du $\mathrm{VI}^{\mathrm{e}}$ et du IVe siècle». Les arguments par lesquels Courby justifiait cette affirmation (la présence, à cet emplacement, d'un «antre » prophétique et d'un édicule très ancien qui aurait été conservé intact à l'intérieur du temple jusqu'au IVe siècle inclusivement) paraissent aujourd'hui discutables, voire erronés, comme on le verra plus loin; mais tout le monde admet, tacitement ou non, que le temple incendié en 548 se trouvait à peu près au même endroit que ses successeurs - et cela est, en soi, extrêmement vraisemblable, même si « rien ne subsiste, sous le temple des $\mathrm{VI}^{\mathrm{e}}$ et IVe siècles, qui ait appartenu à leur prédécesseur, comme constructions du moins» (P. DE LA COSTE-MESSELIĖRE, BCH70 [1946], p. 281. Toutefois, E. HANSEN, FD II 14 [2010], p. 165, attribue au temple du haut-archaïsme "plutôt » qu'au temple des Alcméonides certains tronçons de maçonnerie inclus dans les fondations actuelles du temple; voir, dans le même sens, id., BCH 133 [2009], p. 128-134).

${ }^{9}$ Hérodote, I, 50. 
2. Le temple dit «temple des Alcméonides « (sa construction fut au moins en partie financée par cette grande famille athénienne) succéda au précédent. Il fut édifié (ou seulement terminé ?) et décoré dans les deux dernières décennies du VIe siècle avant notre ère ${ }^{10}:$ il était achevé vers $500^{11}$. Auparavant, on avait procédé à d'importants travaux préliminaires ${ }^{12}$ : extension des limites du sanctuaire de 12 ou $13 \mathrm{~m}$ dans toutes les directions ${ }^{13}$; destruction ou remblaiement de plusieurs édifices (dont les ruines du temple incendié); construction, pour le nouveau temple, d'une vaste terrasse soutenue par le grand mur polygonal resté en place jusqu’à nos jours, etc. Le «temple des Alcméonides » fut détruit à son tour, sans doute dans les années 370 avant notre ère ${ }^{14}$, par une catastrophe naturelle dont la nature exacte est incertaine (glissement de terrain ? séisme? glissement de terrain déclenché par un séisme? accompagné de chutes de rochers ?) ${ }^{15}$. Beaucoup de

10 Très probablement entre 513 et 505, comme l'a montré Th. Homolle, BCH 26 (1902), p. 597-627, dans un article fondamental où tous les textes sont cités, traduits et méthodiquement comparés, analysés et discutés. Cependant, Th. Homolle pensait qu'entre 513 et 505, les Alcméonides avaient seulement achevé à leurs frais la construction du temple et pourvu à son décor sculpté. F. COURBY, FD II. La terrasse du temple, p. 110-111 (et 317) avait adopté le point de vue de Th. Homolle. Aujourd'hui, conformément aux conclusions de P. DE LA CosTE-MESSELIÈRE, « Les Alcméonides à Delphes ", BCH 70 (1946), p. 271-287, on pense plutôt que le temple a été " bâti et décoré entre 513 et 505 »(P. AmANDRY, Ruine, p. 26 = FD II 14, p. 13; les italiques sont de moi [G. Rougemont]) et que «pour des raisons architecturales, il est peu vraisemblable que les Alcméonides aient seulement achevé un ouvrage déjà commencé (ou du moins qui aurait dépassé le niveau des fondations) » (J.-Fr. BOMmELAER, GDS, p. 183). S’il en est bien ainsi, la construction et le décor sculpté du temple auraient été exécutés en sept ou huit ans seulement.

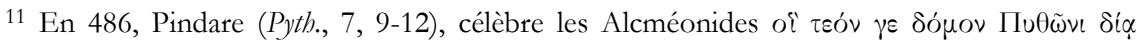

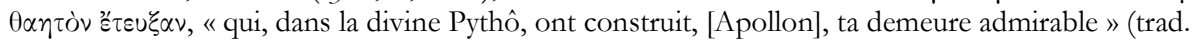

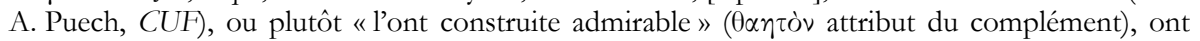
construit ta demeure de façon telle qu'elle mérite d'être contemplée et admirée.

12 Évoqués avec talent par P. DE LA Coste-MESSELIÈre, BCH 70 (1946), p. 279-285.

13 Voir la récente mise au point de J.-Fr. Bommelaer, « Les périboles de Delphes », Pallas 87 (2011), p. 13-38.

14 Il semble qu'en 371, on cherche à collecter de l'argent pour le reconstruire. On tire cette conclusion de Xénophon, Helléniques VI, 4, 2, où, cependant, Delphes n'est pas nommée. Commentaire sur ce texte: G. Roux, L'Amphictionie, Delphes et le temple d'Apollon an IV siècle, Lyon/Paris, 1979, p. 142-146; mais ce commentaire va au-delà des conclusions que le texte autorise et, en tout cas, le décret athénien de 368 utilisé par G. Roux, o.c., p. 146, ne concerne probablement pas le temple de Delphes. Les documents épigraphiques trouvés à Delphes et relatifs au financement de la reconstruction (infra, note 17) permettent de faire remonter les débuts de ce financement à 366 - date certaine, celle-ci (J. BOUSQUET, ECD, p. 24 et CID II, p. 6). La date de 373 traditionnellement attribuée à la catastrophe et reproduite sans discussion par E. HANSEN, FD II 14 (2010), p. 147, est une hypothèse vraisemblable: c'est la date, bien attestée, de séismes qui provoquèrent de graves destructions sur les rives du golfe de Corinthe. Mais aucun texte n'établit de lien entre ces séismes et la destruction du temple de Delphes. En fait, la seule mention directe de cette destruction se trouve dans une inscription mutilée (ChoixInscrDelphes, $\mathrm{n}^{\circ}$ 36) qui n'enseigne rien de sûr ni sur la date, ni sur les causes de l'événement. Résumé de la question : P. SÁNCHEZ, L'Amphictionie des Pyles et de Delphes, Stuttgart, 2001, p. 125 et note 4. Voir aussi la note suivante.

15 J. BousQueT, ECD, p. 19 note 4 : «La catastrophe elle-même doit être due à un séisme, qui a provoqué un glissement de terrain et a détaché des Phédriades une avalanche de pierres de toute taille» (suit un développement détaillé, avec examen de l'ensemble du site). E. HANSEN, 
blocs extraits de la ruine furent aussitôt réutilisés, soit dans les fondations du temple suivant, soit dans les murs de soutènement construits ou reconstruits aux alentours. Grâce à ceux de ces blocs qui sont accessibles à l'étude ${ }^{16}$, on connaît avec certitude le plan d'ensemble, les dimensions, les proportions du temple des Alcméonides; en revanche, son aménagement intérieur reste inconnu. Or ce temple est celui qu’ont connu Eschyle, Pindare, Hérodote, Euripide - le seul qu'ils aient connu.

3. Le «temple du IV siècle», construit pour remplacer le «temple des Alcméonides », fut achevé vers 330 : la reconstruction (y compris les travaux préalables de déblaiement, de consolidation, de construction des soutènements, etc.) avait donc occupé près de quarante ans - avec une interruption de plusieurs années pendant la troisième guerre sacrée ${ }^{17}$. Ce temple subit ensuite, au cours des siècles, divers dommages suivis de réparations, mais il resta debout jusqu'à l'Antiquité tardive ${ }^{18}$. Il ne fut pas transformé en église. À une date impossible à

FD II 14, p. 145-155, entend montrer « qu'il s'est agi pour l'essentiel d'un glissement du sol causé soit par un phénomène séismique, soit par la circulation des eaux souterraines qui ont pu mettre en déséquilibre le manteau hétérogène de pierraille et d'argile qui constitue le soubassement immédiat de Delphes et repose en discontinuité sur le socle de calcaires massifs, mais fracturés du Parnasse ». Il reste nécessaire de commencer l'étude de cette question par la lecture de F. COURBY, FD II. La terrasse du temple, p. 112-113.

16 Ils ne le sont pas tous, loin de là : pour qu'ils le soient, il faudrait démonter en grande partie, voire en totalité, non seulement plusieurs murs de soutènement antiques dont la présence est encore aujourd'hui nécessaire pour empêcher les glissements de terrain, mais aussi les fondations mêmes du temple, et disposer sur le terrain des espaces nécessaires pour étudier commodément tous ces blocs.

${ }^{17}$ Les travaux de reconstruction et leur financement sont documentés par un riche ensemble d'inscriptions, les «comptes de Delphes». Voir les exposés synthétiques de Fr. LEFÈVRE, L'Amphictionie pyléo-delphique, Athènes/Paris, 1998, p. 257-268, et de P. SÁNCHEZ, o.c. (n. 14), p. 124152, rendus possibles par le travail préalable de deux ou trois générations de savants sur ces textes très difficiles, notamment par les travaux fondamentaux de J. Bousquet. Ceux-ci sont disponibles en deux volumes : J. BOUSQUET, CID II. Les comptes du IV et du III siècle, Athènes/ Paris, 1989, où tous les documents sont rassemblés, traduits et commentés, et J. BOUSQUET, Études sur les comptes de Delphes, Athènes/Paris, 1988, à consulter dans cet ordre; une conférence constitue par ailleurs son exposé synthétique le plus accessible: «La reconstruction du temple d'Apollon à Delphes au IV siècle avant Jésus-Christ», in D. KNOEPFLER (éd.), Comptes et inventaires dans la cité grecque, Neuchâtel/Genève, 1988. G. Roux a développé des thèses différentes de celles de J. Bousquet sur quelques points importants (le nombre et la fonction des prytanes, la chronologie des phases de la reconstruction du temple) : voir essentiellement son livre L'Amphictionie, Delphes et le temple d'Apollon au IV siècle, Lyon/Paris, 1979, et dans Gnomon 62 (1990), p. 325-330, son compte rendu de l'ouvrage de J. Bousquet, ECD. Depuis la publication des travaux de J. Bousquet et de G. Roux, les travaux plus anciens (notamment ceux d'É. BourgueT, FD III 5, et de H. POMTOw, Syll.3 236-253) sont, pour l'essentiel, périmés. Travaux plus récents: P. MARCHETTI, Topoi 8 (1998), p. 167-172; id., BCH 123 (1999), p. 405-422; id., BCH 126 (2002), p. 59-72. Sur les artisans employés sur les chantiers du temple, leurs statuts, leurs tâches, leurs salaires, voir Chr. FEYEL, Les artisans dans les sanctuaires grecs aux époques classique et hellénistique, à travers la documentation financière, Athènes/Paris, 2006, et E. Hansen, in P. Amandry et E. Hansen, FD II. Le temple d'Apollon du IVe siècle, Athènes/Paris, 2010, p. 461-465, 490-494, et passim.

18 Ces dommages et ces réparations - du moins ceux et celles qui sont attesté[e]s par les documents parvenus jusqu'à nous... - ont été récapitulés et étudiés par AMANDRY, Ruine, 
fixer avec précision, il fut méthodiquement démonté pour permettre la récupération des agrafes de bronze qui liaient les blocs entre eux. Presque partout ce démontage fut poussé jusqu'aux fondations: en quelques endroits seulement le dallage est encore en place ${ }^{19}$. Jusqu'à la fin du XIX siècle, les maisons d'un village ont occupé l'emplacement du sanctuaire antique ${ }^{20}$, donc aussi l'emplacement du temple ${ }^{21}$. Ce sont les ruines du «temple du IVe siècle» qu'on peut voir aujourd'hui, partiellement restaurées. C'est ce temple que Pausanias a visité22. C'est

particulièrement p. 36-47 = FD II 14, p. 18-23 : incendie causé par les Maides (?), peuple thrace, vers l'époque de la guerre de Mithridate; réfection sous Domitien, aux frais de l'empereur (ChoixInscrDelphes, $\mathrm{n}^{\circ}$ 229); réfection sous les Sévères, par les soins du proconsul d'Achaïe Cn. Claudius Leonticus (ChoixInscrDelphes, $\mathrm{n}^{\circ}$ 267); enfin un incendie volontaire de grande ampleur, suivi d'une réfection sommaire. P. Amandry attribue ce dernier incendie aux chrétiens et situe l'événement au IVe siècle, avant l'édit de Théodose. Contra: V. DÉROCHE, « La dernière réparation païenne du temple d'Apollon à Delphes ", in Mélanges Jean-Pierre Sodini, Travaux et Mémoires, Centre de recherche d'histoire et civilisation de Byzance 15 (2005), p. 231-244; V. Déroche est approuvé par E. HANSEN, FD II 14, p. 147 note 2. Sur les causes matérielles de la destruction finale du temple (une "catastrophe soudaine », de même nature que celle qui détruisit le temple des Alcméonides, c'est-à-dire " un glissement du terrain instable sur lequel Delphes est installée », bientôt suivi du démontage de la ruine et de la récupération des scellements), observations précises et hypothèses nouvelles de E. HANSEN, FD II 14, p. 145-147.

${ }^{19}$ Les planches I (photographie) et II (dessin reproduit ici fig. 1) de la publication de F. COURBY, FD II. La terrasse du temple, sont, à mon goût, les documents qui donnent aux non spécialistes l'image la plus parlante de l'état de la ruine avant sa restauration partielle. Celle-ci fut exécutée, pour l'essentiel, entre 1938 et 1950 (sur ces travaux de restauration, bref résumé et références chez E. HANSEN, FDII, 14, p. 125, qui énumère là les autres relevés de la ruine antérieurs à sa restauration).

${ }^{20}$ Après « la destruction de la ville [protobyzantine], destruction dont la date semble coïncider avec celle des invasions slaves de la fin du vie siècle [de notre ère] et du début du VII ${ }^{\mathrm{e}}$, mais dont un séisme peut aussi bien être la cause, (...) des villages faiblement peuplés se sont succédés pendant environ 1300 ans à l'emplacement du sanctuaire d'Apollon, dont les ruines n'étaient pas encore recouvertes complètement par les terres et la pierraille lors du passage de Cyriaque d'Ancône [en 1436], ni même à la fin du [XIX] siècle, quand le village de Castri fut détruit une dernière fois, par les archéologues » (AMANDRY, Ruine, p. $28=$ FD II 14, p. 14). Des vues un peu différentes, fondées sur un raisonnement a silentio à partir du résultat des fouilles récentes, sont développées par Pl. PÉTRIDIS, «Delphes, Castorion, Castri», BCH 127 (2003), p. 301-310, particulièrement p. 303; voir aussi id., FD V 4. La céramique protobyzantine de Delphes (2010), p. 22: «La stratigraphie des fouilles nous montre un abandon soudain survenu dans le premier quart du VII esiècle. Cet abandon n'a pas été suivi d'actes de violence comme des incendies. À partir de ce moment, le manque total de restes architecturaux, de céramique ou de monnaies, à quelques exceptions près, nous amène à penser que la ville protobyzantine de Delphes n'a pas été réoccupée jusqu'au XIII', voire jusqu'au XIVe siècle ». Id., ibid., note $40:$ «Les monnaies franques et vénitiennes enfouies aux XIVe et XVe $s$. sont les seules traces d'occupation avant le passage de Cyriaque d'Ancône qui, en 1436, reconnaît le site antique de Delphes sous le village de Castri ». - Sur la ville protobyzantine (IVe-VII ${ }^{e}$ s.) : P. AMANDRY, BCH 105 (1981), p. 721-740; AMANDRY，Ruine, p. 46-47 = FD II 14, p. 23; Pl. PÉTRIDIS, «Delphes dans l'Antiquité tardive : première approche topographique et céramologique », BCH 121 (1997), p. 681-695; et surtout id., FD V 4. La céramique protobyzantine de Delphes: une production et son contexte, Athènes/Paris, 2010, passim, en particulier p. 17-31 et pl. 1-7.

21 «Le socle du temple en calcaire de Saint-Élie (...) offrait une plate-forme trop unie et trop solidement bâtie pour qu'elle soit restée inutilisée » (AMANDRY, Ruine, p. 47 = FD II 14, p. 23).

${ }^{22}$ Mais il a cru qu'il s'agissait du temple des Alcméonides : «Il était incapable de distinguer des formes d'architecture et des styles de sculpture séparés par un intervalle de près de deux siècles, au 
celui où Plutarque exerça les fonctions de prêtre d'Apollon. C'est le seul des trois temples successifs dont l'aménagement intérieur ne soit pas complètement inconnu ${ }^{23}$. Sur ce point, les recherches archéologiques et architecturales de P. Amandry et E. Hansen ont apporté des données neuves, rassemblées sous leurs deux signatures dans une publication récente ${ }^{24}$.

\section{L'aménagement intérieur du temple du IV siècle : certitudes et probabilités}

Pausanias (X, 24, 1-5), quand il vient à parler de l'intérieur du temple, évoque d'abord ce qu'il a vu dans le pronaos ${ }^{25}$, ensuite ce qu'il a vu dans le naos ${ }^{26}$, enfin

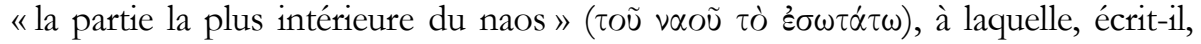

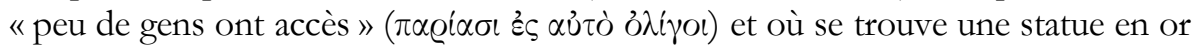
d'Apollon, mais où, semble-t-il, lui-même n'est pas entré27.

cours desquels l'art grec avait évolué dans tous les domaines plus vite et plus radicalement qu'à aucun autre moment de son histoire» (AMANDRY, Ruine, p. 26 = FD II 14, p. 13). Pausanias, j'imagine, se fiait d'abord à ses lectures : les auteurs considérés, de son temps déjà, comme "classiques » ne parlaient guère de la destruction et de la reconstruction du temple au IVe siècle (événement pratiquement révélé aux modernes par la fouille), alors qu'Hérodote, Isocrate, Aristote parlaient en termes clairs du temple des Alcméonides (voir les textes dans l'article de Th. Homolle cité supra note 10$)$.

${ }^{23}$ Mais ne peut-on pas supposer que la disposition intérieure du temple du IVe siècle reproduisait plus ou moins celle des temples antérieurs? - Oui, on peut le supposer. Cette supposition est même vraisemblable. Est-elle vraie ? On ne peut actuellement ni démontrer qu'elle l'est, ni démontrer le contraire. Et puis, tout est dans le "plus ou moins » : il serait étonnant que, d'un temple à l'autre, aucune modification n'ait été introduite - mais sur quels points ? «Il faut bien marquer aujourd'hui la légitimité dans la science du non liquet, et que l'ars nesciendi est un instrument intellectuel indispensable dans la conquête de la vérité, conquête de la vérité et non jeu de bulles de savon » (L. ROBERT, CRAI 1955, p. 200 = Opera Minora selecta I, p. 581).

${ }^{24}$ FD II. Topographie et architecture, 14 : P. AMANDRY et E. HANSEN, Le temple d'Apollon du IVe siècle, 2010; volume I : texte; volume II : planches; volume III : dépliants. Abréviation utilisée dans la présente étude: FD II 14. - Le volume I de cet ouvrage juxtapose a) les articles publiés par P. Amandry sur le temple, b) l'étude architecturale détaillée rédigée par E. Hansen, fruit du travail commun des deux auteurs. On trouvera p. 495-497 ("Épilogue ») une récapitulation des nouveautés apportées par eux et, p. 454 (sous le titre «Une vue d'ensemble»), une description synthétique, illustrée de plans, du temple tels qu'ils se le représentent au terme de leur travail.

${ }_{25}$ Plus exactement, ce qu'il a vu et dont il a choisi de parler: les maximes des Sept Sages, une effigie de bronze d'Homère...

${ }^{26}$ Un autel de Poséidon; un groupe statuaire comprenant les statues de deux Moires et celles

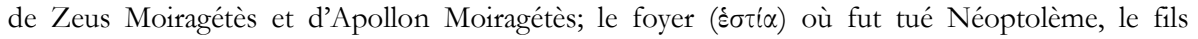
d'Achille; le fauteuil (Ө@óvos) où Pindare s'asseyait quand il venait à Delphes pour composer des hymnes à Apollon.

${ }^{27}$ Comme l'admettent unanimement les commentateurs. C'est en effet l'impression que produit le texte, bien que cela ne soit pas dit expressément. Après avoir parlé de ce qu'il a vu

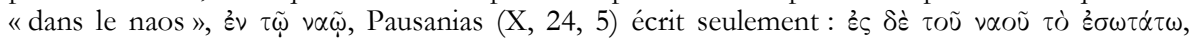

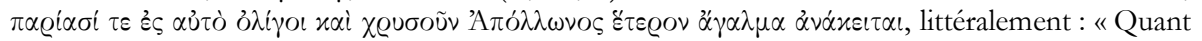
à la partie la plus intérieure du naos, peu de gens y pénètrent et une autre statue d'Apollon, en or, y est consacrée comme offrande. » 
L'étude archéologique et architecturale, quant à elle, nous fait traverser, d'Est en Ouest (Fig. 2), d'abord le pronaos, avec des bancs de pierre ${ }^{28}$, de longues inscriptions ${ }^{29}$ gravées sur les murs à partir du dernier quart du II ${ }^{\mathrm{e}}$ siècle avant notre ère, des scellements dans le dallage au pied des murs ${ }^{30}$, peut-être pour des stèles ${ }^{31}$, puis le naos ou, si l'on préfère le mot latin, la cella, profonde de presque $25 \mathrm{~m}$, pourvue, comme il est habituel, d'une double colonnade intérieure $^{32}$. À l'intérieur du naos, la nef centrale (l'espace compris entre les deux colonnades intérieures), large de plus de 5,60 m, est divisée matériellement en deux parties successives.

a) Quand on vient du pronaos, on rencontre d'abord, sur une profondeur (E-O) de moins de $7 \mathrm{~m}$, un espace entièrement dallé, ouvert au $\mathrm{N}$ et au $\mathrm{S}$ par deux portes latérales ${ }^{33}$ donnant sur la péristasis (c'est-à-dire sous la colonnade extérieure qui enveloppait le temple), et limité vers l'O par une cloison ou une barrière épaisse de $60 \mathrm{~cm}$ environ, scellée sur le dallage, vraisemblablement assez lourde (la fondation du dallage est renforcée à cet endroit) ${ }^{34}$.

${ }^{28}$ E. HANSEN, FD II 14, p. 282-284.

29 P. AmANDry, E. HANSEN, FD II 14, p. 258-265, avec en particulier le dessin restitué fig. 8.3 , p. 263 , déjà publié par D. Rousset (o.c. infra dans la présente note), dont le travail épigraphique a contribué à la restitution proposée dans FD II 14. Quant aux inscriptions, il s'agit, d'une part, des pièces d'un procès amphictionique relatif à des malversations dans la gestion des biens du dieu, avec une délimitation complète de la terre sacrée d'Apollon (vers 117 av. n.è.), d'autre part des arbitrages d'un magistrat romain dans un différend frontalier entre la cité de Delphes et ses voisines (sous Trajan, vers 110 de n.è.), avec des textes gravés à la fois en grec et en latin (d'où le nom traditionnel de cet ensemble : «le Monument bilingue de Delphes »). A. Plassart, FD III 4, n 276-284 et 290-296 (1970) a publié ensemble tous ces textes. Les éditions de référence sont aujourd'hui celles de Fr. LEFÈVRE, CID IV (2002), 119 A-H, et de D. Rousset, Le territoire de Delphes et la terre d'Apollon (2002), p. 80-106 et 197-199, mais chacune d'elles ne reproduit qu'une partie des textes : voir G. RougEMONT, Topoi 14 (2006), p. 487-488. A. Jacquemin, D. Mulliez, G. Rougemont, ChoixInscrDelphes, nº 174 à 177 et 246-247, ne reproduisent, eux aussi, qu'une partie du dossier, mais proposent une présentation globale d'après le travail de D. Rousset.

30 P. Amandry, E. Hansen, FD II 14, p. 281-282.

31 Peut-être celles qui portaient les maximes des Sept Sages : AmANDry, Omphalos, p. $179=$ FD II 14, p. 86.

32 Étudiée en détail par E. HANSEN, FD II 14, p. 287-315 et 323-331. L'existence de cette colonnade, attendue dans un temple de cette époque et de ce type, a été reconnue dès 1915 par F. COURBY, FD II. La terrasse du temple, p. 40-43, puis contestée par J. Replat dans les années 1920 et, pour finir, définitivement établie en 1969 par AMANDRY, Temple, p. 14-38 = FD II 14, p. 52-72.

33 Amandry, Cella, p. 276-278 = FD II 14, p. 75-77; E. HANSEN, FD II 14, p. 270-275. - Le temple de Delphes est orienté NE-SO; pour des raisons de commodité, on s'exprime habituellement comme s'il était orienté exactement E-O.

34 À propos de cette cloison, puis de tout l'espace compris entre cette cloison et le fond de la cella (espace où devait se trouver l'adyton), je renvoie une fois pour toutes aux observations et aux raisonnements d'AMANDRY, Cella (particulièrement p. $276=$ FD II 14, p. 75 [la cloison] et p. 277-281 = FD II 14, p. 77-79 [l'espace au fond de la cella]) et d'E. HANSEN, FD II 14, p. 333387. La conclusion détaillée d'E. HANSEN, o.c., p. 385-387 peut suffire au lecteur non spécialiste de l'architecture grecque. 
b) Plus à l'O, au-delà de cette cloison, sur une profondeur (E-O) d'environ $18 \mathrm{~m}$, s'étendait au fond du temple un vaste espace dans lequel il faut nécessairement situer l'adyton, le lieu où la Pythie prophétisait. Or cet espace n'était que partiellement dallé. Aussitôt à l'O de la cloison, dans la nef centrale, sur une longueur (E-O) de 7 à $8 \mathrm{~m}$, une sorte de "trottoir» dallé s'étendait le long de la colonnade intérieure $\mathrm{N}$ et un autre dallage analogue s'étendait le long de la colonnade intérieure S. Le «trottoir» $\mathrm{N}$ était plus large, plus épais et plus solidement fondé que le «trottoir» $\mathrm{S}$. Entre ces deux «trottoirs", au milieu de la nef centrale, il n'existait aucun dallage ${ }^{35}$. Plus à l'O, et jusqu'au fond de la cella, sur une profondeur (E-O) de plus de $10 \mathrm{~m}$, la nef centrale de la cella n'était pas dallée du tout. Les deux nefs latérales $\mathrm{N}$ et $\mathrm{S}$, en revanche, semblent avoir été dallées jusqu'au fond de la cella.

Selon P. Amandry et E. Hansen, divers indices permettent d'affirmer que les espaces non dallés ne l'ont jamais été, que le niveau du sol dans les espaces non dallés n'était pas sensiblement inférieur au niveau des dallages $^{36}$, que ce curieux dispositif a été prévu dès l'époque de la construction du temple, sinon dès le commencement des $\operatorname{travaux}^{37}$, et qu'il n'existait, au fond du temple, aucun local souterrain ${ }^{38}$.

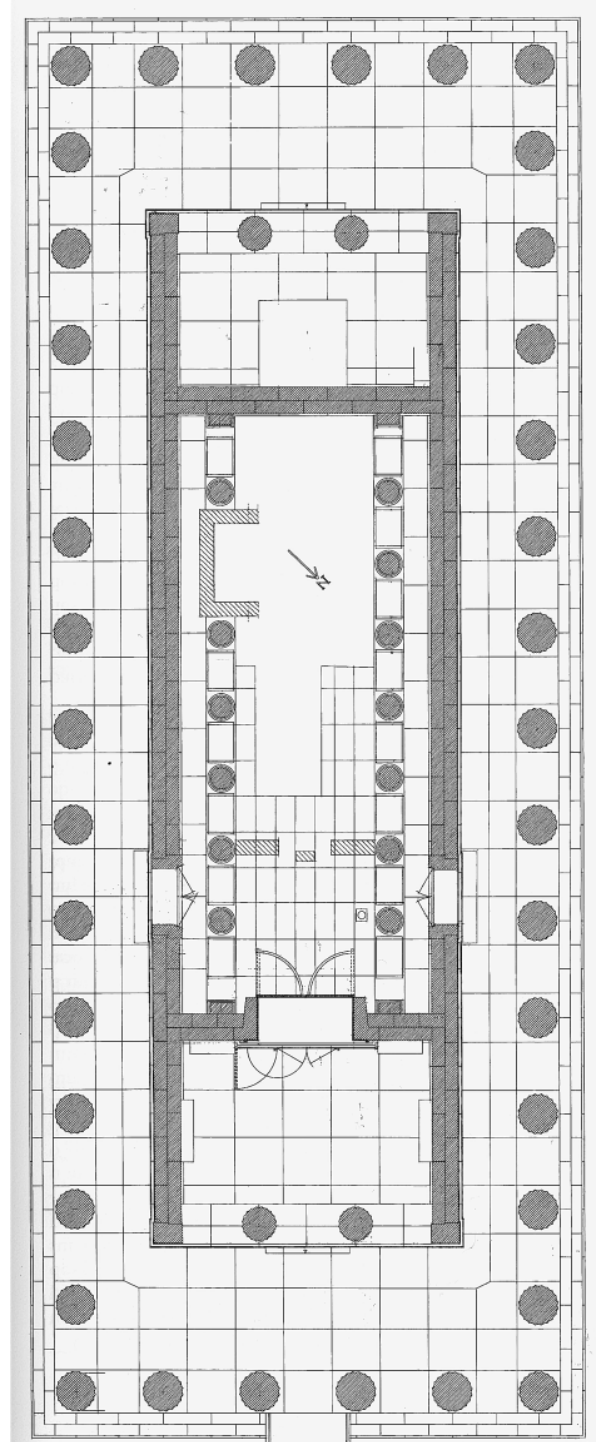

Fig. 2. Plan restitué du temple d'Apollon (E. Hansen, o.c. [n. 24], fig. 18.19).

35 Ce dispositif très particulier (voir, pour d'autres détails, E. HANSEN, FD II 14, p. 386, "Anomalies, hésitations, changements ", $5^{\circ}$ à $\left.9^{\circ}\right)$ n'a reçu jusqu'ici aucune explication.

${ }^{36}$ En effet, « il est peu probable que les fondations du dallage aient été visibles » (AMANDRY, Cella, p. $279=$ FD II 14, p. 78).

${ }^{37}$ Sur cette restriction, voir infra, «Incertitudes et divergences ». 
En tout cas, l'emplacement présumé de l'adyton n'était occupé, au moment de la fouille, par aucun vestige en place. Cette région du temple a donc pu être explorée jusqu'au roc vierge. Elle l'a été à plusieurs reprises ${ }^{39}$. On n'y a trouvé ni source souterraine, ni adduction d'eau amenant dans l'adyton l'eau d'une source extérieure au temple; on n'a pas trouvé trace non plus d'un système qui aurait permis de puiser, dans l'adyton, l'eau d'une nappe souterraine; on n'a trouvé que du remblai, comme il est naturel sous un édifice important. Sur le roc vierge, il n'y a ni fissure ni crevasse. D’ailleurs, il est géologiquement invraisemblable qu'une crevasse aujourd'hui refermée ait jamais existé à cet endroit et qu'il y ait jamais eu à Delphes des émanations volcaniques.

Dans l'opisthodome du temple, enfin, une grosse base carrée avait été scellée sur le dallage, dans l'axe E-O du temple, adossée à la face extérieure du mur $\mathrm{O}$ de la cella, et cela, semble-t-il, dès la construction du temple ${ }^{40}$.

Telles sont, sommairement résumées, les conclusions auxquelles se sont arrêtés P. Amandry et E. Hansen. Leur travail repose sur le catalogage exhaustif et l'étude méthodique, bloc par bloc, de tous les vestiges du temple qu'ils ont pu identifier sur le site - une enquête qu'ils ont été les premiers à mener à son terme. Naturellement, de nombreuses incertitudes subsistent; P. Amandry et E. Hansen ne les dissimulent pas. Et, d'autre part, sur certains points, les deux auteurs ne sont jamais tombés d'accord et la publication ne cache pas leurs divergences. Énumérons incertitudes et divergences - celles, du moins, qui touchent de près à la disposition du local oraculaire.

38 En tout cas il n'existe pas le plus petit indice qui permette d'en restituer un. «Nous sommes obligés d'imaginer un sol en terre battue, à un niveau de peu inférieur à celui du dallage » conclut E. HANSEN, FD II 14, p. 386. «L'aspect des fondations parait exclure qu'une dénivellation importante ait jamais existé » écrit AMANDRY, Cella, p. 281 (= FD II 14, p. 79), qui cite E. Bourguet, Les ruines de Delphes, Paris, 1914, p. 249 : «Les murs [du local souterrain supposé], qui sont simplement les fondations de la colonnade intérieure, ont-ils jamais eu un revêtement? Il n'en reste rien ». P. Amandry conclut alors : «Parce que ce revêtement n'a jamais existé, pas plus que la salle souterraine dont il aurait garni les murs ». C’est bien là, en effet, la façon la plus simple et la plus naturelle de rendre compte des données archéologiques.

39 «Ne trouvant rien au niveau du dallage antique, et convaincus par l'exégèse généralement admise de quelques textes grecs et latins que les oracles étaient rendus dans une salle souterraine qui s'emplissait de l'odeur des émanations du $\chi \dot{\alpha} \sigma \mu \alpha \gamma \tilde{\eta} \varsigma$, les fouilleurs mirent à profit l'absence de restes antiques dans la partie occidentale de la cella, sur une dizaine de mètres de longueur, pour pousser les fouilles en profondeur jusqu'au pied des fondations des murs, et même plus bas. Ils atteignirent des rochers et une nappe d'eau, sans rencontrer la moindre trace d'installation humaine " (AMANDrY, Cella, p. $271=$ FD II 14, p. 73). Les photographies rassemblées par E. HANSEN, FD II 14, p. 317-319, donnent une image tout à la fois impressionnante et pittoresque de ces explorations toujours recommencées.

40 Amandry, Omphalos; Amandry, Opisthodome; E. Hansen, FD II 14, p. 285-286. 


\section{L'aménagement intérieur du temple du IV siècle : incertitudes et divergences}

De la «cloison » qui limitait vers l'O la première partie (dallée) de la cella, « on ne peut pas dire s'il s'agissait d'une simple barrière ou d'un mur d'une certaine hauteur, ni s'il s'étendait sur toute la largeur de la cella ou si on pouvait le contourner par les nefs latérales. Il y avait assurément en son milieu une ouverture large, mais nous ne savons pas si elle pouvait être fermée par une porte ou éventuellement par un rideau. En tout cas, le passage semble avoir été partiellement obstrué dans sa partie centrale par un objet scellé au sol : base, piédestal, autel ? ${ }^{41}$ »

P. Amandry et E. Hansen affirment que l'aménagement de la partie occidentale de la cella remonte à l'époque de la construction du temple; mais ils ont observé les signes de changements intervenus au cours de la construction, ainsi que diverses «anomalies» ou «hésitations» dans l'exécution du programme. «Sans aucun doute, la division de la cella en trois nefs a été prévue dès le début (...), mais la construction des fondations en poros des colonnades n'a apparemment pas été commencée avant l'achèvement des fondations en poros du sèkos, car il n'y a pas de liaison entre ces deux parties de la construction et la hauteur des assises est différente $»^{42}$. «Les trois assises supérieures (...) des substructions des colonnades [intérieures], aussi bien au Sud qu'au Nord, présentent vers la nef médiane des faces bien parées, qui font penser qu'elles étaient destinées à être visibles au-dessus d'un sol plus bas ou, du moins, qu'une décision n'était pas encore prise au moment de leur pose (...). Or, finalement, ces constructions furent entièrement cachées $«{ }^{43}$. « Ni dans la taille ni dans la pose des pierres du stylobate [de la colonnade intérieure], ni dans leur liaison à l'assise inférieure, ni dans le ravalement final, on ne retrouve le principe de régularité presque mécanique qui a présidé à la construction du temple dans toutes ses autres parties. On devine que ces légères anomalies découlent des servitudes qu'imposaient aux entrepreneurs l'aménagement de la cella en siège d'un oracle $»^{44}$. «Cet ensemble d'observations donne l'impression de multiples hésitations de la part des constructeurs confrontés à un aménagement intérieur de la cella qui n'était pas strictement prévu dès le début des travaux, probablement parce que le fonctionnement particulier du lieu sacré posait des problèmes inhabituels $»^{45}$.

${ }^{41}$ E. Hansen, FD II 14, p. 385.

42 E. HANSEN, FD II 14, p. 287.

${ }^{43}$ E. HANSEN, FD II 14, p. 386; voir déjà AMANDry, Temple, p. 682 et fig. 6 = FD II 14, p. 31 et p. 33, fig. 2 .

44 Amandry, Temple, p. 37-38 = FD II 14, p. 72.

${ }^{45}$ E. Hansen, FD II 14, p. 387. 
E. Hansen pense que, dans l'adyton, la colonnade intérieure $S$ s'interrompait sur une longueur de quelques mètres et que, dans l'espace ainsi laissé libre, se dressait un petit édifice (un oikos, disent les savants modernes), sans doute plus ancien que le temple, édifice que les constructeurs du temple du IV siècle avaient dû intégrer tel qu'il était, sans pouvoir ni le déplacer, ni le détruire. C'était là l'hypothèse présentée par F. Courby ${ }^{46}$. E. Hansen la reprend à son compte et la défend avec une argumentation développée ${ }^{47}$. Il est incontestable qu'au moment où la ruine du temple fut déblayée par les fouilleurs, la fondation de la colonnade intérieure $S$ (le seul vestige de cette colonnade qui fût encore en place) était matériellement interrompue et que, à cet endroit précis, quelques blocs de poros ${ }^{48}$ pouvaient suggérer la présence d'un édicule de plan carré. Pourtant, l'hypothèse de F. Courby, adoptée par E. Hansen, inspirait à P. Amandry un scepticisme manifeste : il la présentait, au mieux, comme une théorie à confirmer ${ }^{49}$ et il avait tendance à voir dans cet étrange dispositif le résultat de remaniements très tardifs ${ }^{50}$. De toute façon, même si cet édicule

${ }^{46}$ F. COURBY, FD II. La terrasse du temple, p. 47-56.

47 FD II 14, p. 296-312, «L'interruption de la colonnade Sud » (conclusion, p. 312 : « Nous devons conclure que l'interruption de la colonnade faisait partie du projet initial »), et p. 315-316, «Y avait-il un "oikos" dans l'adyton?». P. 315: «Le tronçon Ouest (de la fondation de la colonnade intérieure Sud) a montré indiscutablement que le stylobate s'arrêtait après la première colonne et qu'il y avait là les fondations d'un mur transversal qui se prolongeait, le long du côté Sud de la cella, jusqu'à un mur dont on reconnaît encore les traces. Les fondations du tronçon Est du stylobate s'arrêtent également contre un bout de mur transversal, mais d'un autre caractère (...) ». P. 316, E.H. envisage comme possible ou probable « une niche de faible profondeur».

48 Qui se sont écroulés quelques années après leur découverte : ils étaient encore en place en 1899, mais déjà F. Courby, dont la publication a paru en 1915, n’a pu les étudier que sur des photographies prises aussitôt après la fouille, photographies qu'il présente et commente dans sa publication FD II. La terrasse du temple, p. 48-50, et que Hansen reproduit à son tour, FD II 14, p. 310-312 (ce sont les plaques photographiques A 168, A 169 et A 170 de la "grande fouille » de Delphes, conservées à la photothèque de l'École française d'Athènes). Avec les blocs de pôros s'était effondré le « tronçon Ouest des substructions de la colonnade Sud ».

49 «La question est de savoir si cette interruption faisait partie du plan primitif, ou si elle résulte de transformations ou d'aménagements exécutés à une date quelconque au cours de plus de 700 ans où le temple a été en service, ou même plus tard, quand le christianisme a supplanté le culte d'Apollon, ou plus tard encore, quand des maisons ont été bâties sur la terrasse du temple. Des pierres portent des traces de retaillages et de mutilations apparemment intentionnelles » (AMANDry, Cella, p. $280=$ FD II 14, p. 78).

50 «C'est à cette période [postérieure à la disparition de la ville protobyzantine (voir ci-dessus la note 20)] que je rapporterais volontiers certains aménagements dont on essaye sans succès depuis près d'un siècle de comprendre le rôle dans la disposition du local oraculaire : interruption par un retaillage grossier du soubassement de la colonnade intérieure Sud (...), construction de petits murs faits de blocs de poros de remploi si mal fondés qu'ils se sont écroulés peu de temps après avoir revu le jour. On a oublié que la vie ne s'était pas arrêtée à Delphes avec la dernière consultation de la Pythie » (AmAndry, Ruine, p. $47=$ FD II 14, p. 23). Voir supra les notes 20 et 21. - Je dois dire qu'un examen superficiel de l'endroit où s'interrompt la fondation de la colonnade intérieure Sud et, surtout, les photographies prises au moment de la fouille incitent à partager les doutes de P. Amandry. Ces photographies (FD II 14, p. 310-312, fig. 11.18 à $11.20=$ F. Courby, FD II. La terrasse du temple, p. 48-50, fig. 46-48, ou encore FD II 14, p. 317 en haut = 
(espace intérieur : 3,25 m de côté) existait dans l'Antiquité, on ne sait rien de ce qu'il pouvait abriter, contenir ou protéger ${ }^{51}$.

Dans le temple tel qu'il s'offre à nos yeux aujourd'hui, le trépied sur lequel s'asseyait la Pythie n'a laissé aucune trace. Le laurier non plus, évidemment. À vrai dire, la présence d'un laurier dans l'adyton est douteuse : les textes, sur ce point, ne sont pas aussi clairs qu'on l'a $\mathrm{cru}^{52}$ et les représentations figurées ne prouvent rien $^{53}$. Or personne n'a jamais expliqué comment un laurier pouvait vivre dans la pénombre de l'adyton et les spéculations sur l'existence, dans la toiture, d'une ouverture plus ou moins importante qui aurait laissé pénétrer la lumière du jour, ces spéculations sont, à ce jour, invérifiables ${ }^{54}$. Quant à l'omphalos, « nombril du monde », on en connaît assez bien l'aspect, grâce aux représentations et aux copies antiques de l'objet, et l'on est sûr qu'il était dans le temple. Mais à quel endroit du temple? Dans l'adyton, comme le pensent la plupart des commentateurs? Dans le pronaos, comme le supposent J. Bousquet et E. Hansen ${ }^{55}$ ? Ou dans l'opisthodome, sur la grosse base carrée adossée au mur qui sépare l'opisthodome de la cella, comme le suggère P. Amandry ${ }^{56}$ ? Cette dernière hypothèse est, actuellement, la plus solidement argumentée des trois, mais elle soulève des objections, comme les deux autres, et elle doit rester une hypothèse, d'après P. Amandry lui-même.

\section{Conclusions}

1. Archéologiquement, on ignore absolument tout de l'aménagement intérieur du temple d'Apollon avant le IV siècle : l'adyton au temps de Crésus, au temps de Pindare et d'Eschyle, au temps d'Hérodote et d'Euripide, peut faire l'objet de conjectures fondées sur les sources écrites et certaines de ces conjectures peuvent être exactes, mais l'archéologie, pour le moment, ne permet ni de les confirmer, ni de les infirmer.

F. COuRBY, o.c., p. 46, fig. 45; le beau dessin de CourBy, o.c., p. 51, fig. 49, est un dessin reconstitué) donnent l'impression, pour le moins, que ce coin du temple avait subi quelques sérieux remaniements depuis la fin de l'Antiquité. Autrement dit, je crois que, s'il s'était agi de n'importe quel temple autre que le temple d'Apollon à Delphes, l'opinion de P. Amandry eût été adoptée sans résistance. Mais enfin je ne possède ni les connaissances, ni les compétences, ni l'expérience nécessaires pour critiquer la démonstration d'E. Hansen, ni celle de F. Courby.

${ }^{51}$ Ce qui, certes, n'interdit pas de faire des conjectures - mais fondées sur quoi ?

52 P. AmANDry, La mantique apollinienne à Delphes, Athènes/Paris, 1950, p. 133-134.

53 Voir les réflexions de méthode de Fr. LissarRague, BCH Suppl. 36 (2000), p. 56.

54 Naturellement, comme beaucoup de spéculations invérifiables, celles-ci sont inattaquables : on ne peut démontrer ni qu'elles sont vraies, ni qu'elles sont fausses. « Même une ouverture vers le ciel ne serait pas impossible » écrit E. HANSEN, FD II 14, p. 440.

${ }^{55}$ E. HANSEN, FD II 14, p. 451 et n. 32.

56 AmANDry, Omphalos et AMANDry, Opisthodome, où l'on trouvera toute la bibliographie antérieure et une discussion approfondie des données du problème et des diverses solutions proposées. 
2. À partir du IV siècle ${ }^{57}$, la cella du temple de Delphes - en tout cas sa nef centrale - était divisée en deux parties. La première, dont on peut supposer qu'elle était accessible à tous, ouvrait sur l'extérieur non seulement par l'entrée principale du temple, mais aussi par deux portes qui donnaient sur les côtés du temple. La seconde partie de la cella, - celle, suppose-t-on, où « peu de gens pénètrent» (Pausanias supra n. 27), celle, en tout cas, où se trouvait l'adyton était séparée de la première par une sorte de cloison ou de barrière, dont on ignore si elle était assez haute pour arrêter la vue. Selon toute probabilité, cette seconde partie de la cella n'était que partiellement dallée, mais son sol de terre battue était au même niveau que les dallages du temple: l'adyton n'était pas souterrain. Enfin, aucune adduction d'eau n’y pénétrait.

Georges ROUGEMONT

Maison de l'Orient et de la Méditerranée Jean-Pouilloux

7 rue Raulin, F- 69007 LYON

Courriel:georges.rougemont@mom.fr

\section{Abréviations}

AMANDRY, Cella

AMANDRY, Omphalos

AMANDRY, Opisthodome

AMANDRY, Ruine

AmAndry, Temple

ChoixInscrDelphes

CID

ECD

FD II 14

GDS
P. AMANDRY, "Recherches sur la cella du temple de Delphes », in J.-G. HeinTz (éd.), Actes du colloque Oracles et prophéties dans l'Antiquité, Strasbourg 15-17 juin 1995, Paris, 1997 (Travaux du centre de recherche sur le Proche-Orient et la Grèce antiques, 15), p. 272-282 = FD II 14, p. 73-84.

P. AmANDrY, "Où était l'omphalos ? ", in J.-Fr. BOMmELAER (éd.), Delphes, centenaire de la "grande fouille » réalisée par l'École française d'Athènes (1892-1903), Actes du colloque Paul Perdrizet, Strasbourg, 6-9 novembre 1991, Leiden, 1992 (Travaux du centre de recherche sur le Proche-Orient et la Grèce antiques, 12), p. 177-205.

P. AMANDRY, "Notes de topographie et d'architecture delphiques. IX. L'opisthodome du temple d'Apollon », BCH 117 (1993), p. 263-283.

P. AMANDRY, «La ruine du temple d'Apollon à Delphes «, Bulletin de la classe des Lettres et des Sciences morales et politiques (Académie Royale de Belgique) 75 (1989), p. 26-47 = FD II 14, p. 13-29.

P. AmANDRY, « Notes de topographie et d'architecture delphiques. V. Le temple d'Apollon », BCH 93 (1969), p. 1-38 = FD II 14, p. 41-72.

A. Jacquemin, D. Muldiez, G. Rougemont, Choix d'inscriptions de Delphes, Athènes/Paris, 2012.

Corpus des Inscriptions de Delphes.

J. BOusQueT, Études sur les comptes de Delphes, Athènes/Paris, 1988.

Fouilles de Delphes II. Topographie et architecture, 14: P. AMANDRY et E. HAnsen, Le temple d'Apollon du IV siècle, Athènes/Paris, 2010; volume I : texte; volume II : planches; volume III : dépliants.

J.-Fr. Bommelaer, dessins de D. Laroche, Guide de Delphes. Le site, Athènes/Paris, 1991.

${ }^{57}$ Et peut-être plus tôt, mais nous n'en savons rien. 